\title{
Ontología social y práctica jurídica: la actualización de un debate
}

\author{
Social Ontology and Legal Practice: \\ Updating a Debate
}

Pablo A. Rapetti*

Recepción: 10/05/20

Evaluación: 31/07/20

Aceptación final: 15/08/20

\begin{abstract}
Resumen: En el número XIV de Discusiones se produjo un debate acerca del rol de los hechos sociales como fundamento ontológico de la práctica jurídica. En las líneas que siguen intentaré reconstruir los elementos centrales de aquel debate sometiéndolos a escrutinio crítico y, sobre todo, intentaré poner en relación las cuestiones allí discutidas con algunos avances que se han dado en la literatura especializada con posterioridad. De esta manera, la finalidad principal del trabajo es la de sugerir algunas vías con las cuales continuar y profundizar la discusión sobre el tema.

Palabras clave: práctica jurídica; ontología; fundamentación; anclaje; Austin, Hart.
\end{abstract}

\begin{abstract}
In Discusiones, XIV, a debate took place about the role social facts play as the ontological grounds of the legal practice. In what follows, I will try to reconstruct the central tenets of that debate, critically scrutinizing them and, more importantly, I will try to trace a link between the issues
\end{abstract}

Doctor en Derecho, Universitat de Girona, España. Profesor del ITAM (Instituto Tecnológico Autónomo de México), Ciudad de México. Contacto: pablo.rapetti@itam.mx. Agradezco a Riccardo Guastini, Guilherme Vasconcelos y la revisión anónima provista por Discusiones las lecturas y observaciones hechas a la primera versión de este trabajo. 
Pablo A. Rapetti

then discussed and some developments worked out in the specialized literature afterwards. Thus, the main goal of this paper is that of suggesting some lines along which to continue and sharpen the debate on the subject. Keywords: legal practice; ontology; grounding; anchoring; Austin; Hart.

La fórmula radical, íntima, de lo que Él estaba haciendo miserablemente, era la ambición y ansiedad de lograr el reemplazo por la Nada de la Totalidad, de todo lo que hay, lo que hubo, lo que es, de toda la Realidad material y espiritual. Creía que el Cosmos, lo Real, no podría soportar mucho tiempo...

Macedonio Fernández - Tantalia

\section{Introducción}

En Discusiones XIV se dio un debate entre Carlos Bernal Pulido, Damiano Canale y Richard Ekins acerca de la ontología del derecho. En particular, el debate versó sobre si, y eventualmente cómo, el derecho es o se funda en alguna clase de práctica social caracterizada de un modo más o menos específico, esto es, como un conjunto de acciones intencionales colectivas por parte de agentes humanos. A su vez, ello implicaría - como señala Giovanni Tuzet en su introducción a la discusión (Tuzet, 2014, p. 10) - que el asunto tratado es, en principio, lo que puede llamarse la "ontología-proceso" del derecho y no este (o sus componentes) en tanto que entidad u objeto, es decir, en tanto que "ontología-resultado".

Según creo, se trata de un debate que aún hoy se encuentra en un estadio incipiente dentro de la teoría analítica del derecho. ${ }^{1}$ Los desarrollos en la ontología social analítica más en general, no ligados específicamente al

1 En cambio, Tuzet parece insinuar, mediante un ejemplo, que al menos las investigaciones sobre ontología-resultado son frecuentes en la teoría jurídica. Según apunta, la pregunta “¿Qué es una norma jurídica válida?” es propia de una investigación de dicho tipo (2014, p. 11). Me parece que este no es necesariamente el caso: aunque es una cuestión discutida, muchos han querido separar las cuestiones de la validez de las normas de aquellas relativas 
derecho, se encuentran en un no muy menor grado de novedad. Con esto no insinúo que la teoría del derecho vaya siempre necesariamente a la zaga de otras disciplinas filosóficas, importando recursos desde ellas. En muchas ocasiones, la novedad puede, a la inversa, "provenir" de la propia teoría del derecho. Y esto se evidencia justamente en el contexto de las actuales investigaciones de ontología social analítica, donde, e. g., los aportes de Hart acerca de la idea de una regla de reconocimiento [en adelante, $\mathrm{RR}$ ] y la relación que esta tiene con ciertos tipos específicos de prácticas sociales - noción de aceptación mediante- tienen carácter precursor. ${ }^{2}$

En todo caso, los objetivos de las líneas que siguen son los de (1) reconstruir el debate Bernal-Canale-Ekins intentando (2) ponerlo en relación con algunas elaboraciones relacionadas que se han producido con posterioridad a dicha discusión y (3) abriendo nuevos frentes de detalle para una posible profundización.

Como bien señala Tuzet (2014, pp. 11-12), la herramienta teórica central del artículo inicial, de Bernal, es la tesis de la práctica social [de aquí en más, TPS]. De acuerdo con esta tesis, el derecho, entendido como un conjunto de normas válidas, está fundado en una práctica social.

La estrategia de Bernal consiste en asumir TPS como intuición de base acerca del derecho y testear en función de ella las concepciones generales del derecho de tres autores: Austin, Hart y Shapiro. Su objetivo es el de comprobar si se trata de concepciones compatibles con la verdad de TPS o, dicho en otros términos, el de ver si son concepciones que den cuenta del derecho en tanto que práctica social. En general, el resultado del examen es que los primeros dos no lo consiguen, mientras que Shapiro, con su teoría del derecho como planificación, sí lo logra. En este balance no me enfocaré sobre la discusión acerca de esta última. Ello se debe en parte a que la réplica de Bernal a las críticas hacia Shapiro es muy escueta y en parte a

a su existencia. Si la distinción resulta plausible, las cuestiones de existencia serán, en efecto, materia de investigación ontológica; pero en cambio las de validez serán, más bien (o, cuando menos: posiblemente), de índole lógica o normativa. Es notorio que las investigaciones sobre validez son bastante más frecuentes que aquellas sobre existencia. Sobre la distinción entre existencia y validez de normas véase, e. g., Guastini (2014, caps. VIII, XII y XIII).

2 Bernal reconoce esto en su réplica a las críticas (Bernal Pulido, 2014b, p. 147). Véase también Roversi (2018, p. 139). 
que el debate en torno a las teorías de Austin y, sobre todo, Hart, permite por sí solo introducir los que creo son los más importantes elementos de actualización del análisis socio-ontológico respecto del derecho.

Vale destacar que, según la réplica de Bernal a las críticas, su estrategia ni presupone la verdad de TPS, ni afirma que las teorías de Austin y Hart, en la medida en que no casan con TPS, sean incorrectas (Bernal Pulido, 2014b, pp. 146-148). Ciertamente, esto no resultaba del todo claro en su artículo original y por ello Damiano Canale le objetaba que, del hecho de que las teorías de Austin y Hart no se condigan con TPS, no se sigue que sean incorrectas, al tiempo que la relación entre TPS y dichas teorías no dice nada acerca de la verdad o falsedad de TPS (Canale, 2014, p. 76).

\section{Acción colectiva vs. agencia grupal}

De acuerdo con Bernal, TPS deriva de algunas intuiciones más básicas. La intuición fundamental es que la existencia de las actividades jurídicas depende de la capacidad que los seres humanos tenemos para actuar de forma colectiva, es decir, como miembros de grupos o sujetos plurales. Bernal denomina "socialidad" a dicha actividad colectiva. El ejercicio de esta capacidad nos permitiría crear una parte específica de la realidad, la "social", a la que pertenecerían - entre otros elementos- las instituciones (Bernal Pulido, 2014a, pp. 22-23).

De acuerdo con TPS, el derecho está fundado en una práctica social. Por su parte, una práctica social es concebida por Bernal "de manera amplia", como un conjunto de acciones intencionales colectivas recurrentes [en adelante, AICR]. Una AICR es tal que

1) es llevada a cabo por una multiplicidad de agentes actuando conjuntamente como grupo y

2) se la realiza de acuerdo con, y en razón de, intenciones "comunes" o "colectivas" [we-intentions]; lo que a su vez exige cierto conocimiento, por parte de los agentes del grupo, de las acciones e intenciones de los demás miembros (Bernal Pulido, 2014a, pp. 24-27). 
Ontología social y práctica jurídica: la actualización de un debate

Esta caracterización básica genera algunas dudas, puesto que en ningún momento se aclara qué quiere decir que los agentes actúen "conjuntamente como grupo" según la condición (1). Al parecer, lo que Bernal tiene en mente es que así sucede cuando se da la condición (2), pero entonces parece que esa parte de la condición (1) resulta redundante. En cualquier caso, una de las objeciones presentadas por Richard Ekins viene aquí a cuento, porque, según él, Bernal se concentra puramente en las AICR, dejando de lado lo que denomina "agencia grupal", y que podría resultar muy importante para el análisis de los fenómenos jurídicos. La agencia grupal supone una más específica forma de acción colectiva en la que el grupo, se espera, puede actuar como un agente singular, racional y natural (Ekins, 2014, pp. 116-117). Esto implica que los miembros del grupo busquen actuar conjuntamente de manera coherente y consistente, razonando de un modo distinto al propio de la deliberación individual, y eso sería lo que diferencia a la agencia grupal de otras formas posibles de AICR. ${ }^{3}$

Bernal responde objetando la posibilidad de que el acto de un grupo sea considerado el acto de un agente único. Para ello equipara las acciones propias de la agencia grupal, en el trabajo de Ekins, con acciones representativas, esto es, acciones realizadas por alguno(s) miembro(s) de un grupo en nombre de todo el grupo. Y, siguiendo a Kirk Ludwig, propone una explicación "deflacionaria" de la intencionalidad colectiva, de acuerdo con la cual el hecho de que el grupo de personas haga algo consiste en que sus miembros individualmente contribuyan a llevar a cabo ese algo (Bernal Pulido, 2014b, pp. 153-154). ${ }^{4}$ De acuerdo con la propuesta de Ludwig, la acción colectiva puede explicarse en términos de acciones de quienes sean representantes del grupo, acciones que a su vez están respaldadas por otras acciones de los demás miembros. Así, Bernal afirma que

[e]l proyecto de la intencionalidad colectiva, que sostiene que la práctica jurídica puede ser explicada en términos de las acciones individuales y colectivas de los agentes que participan en ella, no

3 En Ekins (2012), el autor busca caracterizar la actividad parlamentaria como propia de la agencia grupal. Su concepción de la agencia grupal viene, al menos en parte, del trabajo de Pettit \& List (2011).

4 Para la presentación original de la idea, véase Ludwig (2013). 
se ve socavado por el fenómeno de la acción representativa y es compatible con el ejemplo en el que la legislatura delega la tarea de expedir leyes y de esta manera la lleva a cabo sin que haya un agente grupal autónomo sino solo individuos que llevan a cabo sus múltiples contribuciones a los actos oficiales del grupo por medio de sus agentes autorizados (Bernal Pulido, 2014b, pp. 155-156).

En este sentido, Bernal parece preocupado por la cuestión estrictamente ontológica de si hay necesidad de postular la existencia de entidades supraindividuales (el grupo en sí). Esta no parece la preocupación que motivó la observación de Ekins, que es más bien la de dar cuenta de acciones individuales de agentes particulares que buscan específicamente la consistencia y coherencia al interior del grupo del que forman parte, acciones alimentadas por un tipo de razonamiento que tiene puntualmente en cuenta la existencia del grupo y la pertenencia a él del propio agente que razona. Según creo, no hay nada en la posición de Ludwig que la muestre prima facie incapaz de ofrecer una adecuada explicación del rasgo que preocupa a Ekins. Pero, en cambio, no parece que Bernal haya elaborado una explicación tal.

Al margen de ello, la cuestión que recién he calificado de "estrictamente ontológica", puede quizás resultar menos problemática de lo que Bernal parece tácitamente creer. Ciertamente, la cuestión de la economía ontológica ha sido considerada relevante al menos desde Quine (2002 [1948]). Cuantas menos entidades presuponga como efectivamente existentes una teoría o construcción explicativa, mejores credenciales tendrá. Por ello la economía ontológica constituye uno de los estándares básicos para la selección entre teorías en competencia. ${ }^{5}$ Sin embargo, en los últimos años la metafísica analítica parece estar dejando de lado este criterio o, quizás, reinterpretándolo: la cuestión ya no se toma tanto en términos de cantidad de entidades, como acaso fue concebida, sino más bien en términos de fundamentalidad. No es per se problemático asumir la existencia de muchas entidades; lo eventualmente problemático es asumir la existencia de muchas entidades en tanto que fundamentales. ${ }^{6}$ Así, e. g., Kit Fine (2001,

5 La presentación más conocida de estos criterios es probablemente la de Thagard (1978). 
2009) distingue entre compromisos ontológicos ligeros y densos. Se tienen compromisos con lo que sea sobre lo que se cuantifique, pero si se cuantifica sobre entidades que están fundamentadas por otras, el compromiso es ligero y poco oneroso. Lo verdaderamente oneroso son los compromisos densos, con entidades fundamentales.

Es igualmente discutido qué entender por "fundamental". Una opción, e. g., consiste en sostener que una entidad no es fundamental en la medida en que puede ser reducida metafísicamente a otra(s). Otra opción consiste en sostener que no es fundamental si está enteramente constituida metafísicamente por otra(s), aún si esto no necesariamente implica que pueda reducirse a esa(s) otra(s). Y, desde luego, hay también diversas alternativas sobre qué suponen los conceptos de reducción y constitución, e incluso sobre si la relación de constitución es solo una o en realidad admite múltiples formas no intercambiables. ${ }^{7}$ Más adelante volveré sobre esto.

\section{Austin y TPS}

La primera teoría del derecho analizada por Bernal (2014a, pp. 22 ss.) es la de John Austin. Para Austin el derecho de una comunidad puede ser entendido como el conjunto de órdenes respaldadas por amenazas de una persona o cuerpo de personas que, al ser habitualmente obedecido sin tener por su parte el hábito de obedecer a nadie más, es entonces el soberano de dicha comunidad. Bernal afirma que la presentación del hábito general de obediencia es ambigua, puesto que admite tanto una lectura distributiva como una colectiva. Bajo la lectura distributiva, cada individuo de la mayoría obediente se conforma a las órdenes del soberano, de manera personal e independiente. Según esta interpretación, dicho hábito es una mera suma de acciones personales. Bajo la lectura colectiva, en cambio, es una AICR.

Si se lee a Austin conforme la óptica colectiva, su teoría parece condecirse con TPS. Es decir, la concepción general que él propone sería una que presenta al derecho como una práctica social. Pero Bernal concluye que, en el mejor de los casos, la teoría de Austin sería una pobre encarnación

7 De manera que estoy usando la palabra "constitución" en sentido no-técnico. 
de TPS, por dos grandes obstáculos. En primer lugar, no es claro que la lectura colectiva sea la que es correcto hacer. En esto consiste el problema que Bernal denomina "de la colectividad". El segundo problema vendría luego, incluso si se asume dicha lectura: "el problema de la normatividad". Bernal afirma ${ }^{8}$ que es un problema que ya Hart había señalado en su clásico examen del trabajo austiniano: la idea del hábito general de obediencia no sería útil para captar

algunas verdades aceptadas sobre la dimensión normativa del derecho, tales como el hecho de que la desviación del comportamiento establecido en las normas produce una reacción crítica y que la gente piensa que dicha reacción crítica está justificada [ni] la verdad aceptada de que la autoridad jurídica no es sólo aquella que tiene la competencia para ejercer la coerción y obligar la sujeción a las normas, sino también "el derecho jurídico para hacerlo"; y que este derecho, o, para ser más precisos, la competencia jurídica para imponer sanciones y la competencia jurídica general para emitir reglas a la población, genera no solo un hábito, sino también una sujeción (en términos de Hohfeld) a obedecer la autoridad jurídica, y la obligación de seguir las reglas jurídicas emitidas por ella (Bernal Pulido, 2014a, pp. 31-32; nota omitida).

Ahora bien, creo que aquí es Bernal quien incurre en una ambigüedad, puesto que no es claro el sentido de "normatividad" - palabra fatigada en la teoría del derecho- que está empleando. Considero que se la suele emplear en al menos tres sentidos distintos. En un primero, la palabra alude al carácter de genuina obligatoriedad que tal vez tenga el derecho, es decir, a la cuestión de si ofrece razones concluyentes para la acción; ${ }^{9}$ lo llamaré normatividad. En otro, a la "forma normativa" del derecho, esto es, al hecho de que este consta estructuralmente de normas (sin juzgar si estas son genuinamente proveedoras de razones concluyentes para la acción); lo llamaré normatividad ${ }_{2}$. Todavía en un tercer sentido, normatividad, alude

8 Equivocadamente, como veremos luego.

9 Normalmente, ello viene acompañado de las asunciones de que se trata de razones morales y de que solo estas pueden ser concluyentes. 
Ontología social y práctica jurídica: la actualización de un debate

al hecho de que los sujetos del derecho de una comunidad consideran que este los obliga, que les brinda razones para actuar.

Me parece indudable que las críticas de Hart a Austin tenían que ver con normatividad y normatividad $_{3}$. No con normatividad ${ }_{1},{ }^{10}$ que es el sentido que preocupa realmente a Bernal (2014a, p. 40). En parte, Canale y Ekins critican también esta inadecuada comprensión de los objetivos teóricos de Hart. Pero ambos parecen endilgar a Hart una preocupación

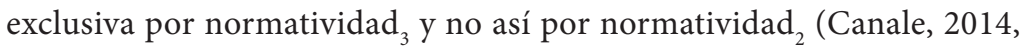
pp. 89-91 y 93-94; Ekins, 2014, p. 130). Ekins agrega -en consonancia con las inclinaciones metodológicas finnisianas que manifiesta- que está de acuerdo con Bernal en que uno no debe quedarse en la exploración de normatividad ${ }_{3}$, insinuando que la teoría del derecho debe afrontar también normatividad $_{1}$ (lo que implica que una teoría del derecho que no lo haga es criticable por ello, como ejemplifica Bernal). Canale (2014, p. 107) parece también compartir esto. ${ }^{11}$ Personalmente, discrepo de los tres y, como Hart y Austin, creo que el análisis de normatividad ${ }_{1}$ es del resorte de las filosofías moral y política. Mientras que normatividad ${ }_{2}$ y normatividad son $_{3}$ abordables desde perspectivas conceptuales y descriptivas (es decir, explicativas), normatividad ${ }_{1}$ resulta propio de una labor justificativa, normativa en sí misma. Y por razones conceptuales: por el concepto mismo de teoría en una versión "restringida", por decirlo de alguna manera. Pero no abundaré sobre esto aquí. ${ }^{12}$

En cambio, me interesa insistir sobre cómo en la disputa no se atribuye a Hart una preocupación por normatividad ${ }_{2}$. Me concentraré solo en un pasaje del texto de Canale, que cito en extenso:

El argumento principal de Hart es que la teoría de Austin acerca del hábito de obediencia no tiene en cuenta la distinción entre el com-

${ }^{10}$ Como veremos a continuación, esto evita la contradicción que Bernal sugiere en la obra de Hart, consistente en que este último señala a Austin el "problema de la normatividad" y, sin embargo, caería también en él.

${ }^{11}$ Sin embargo, sus conclusiones acerca de la obra de Hart (Canale, 2014, pp. 93-94) alimentan dudas sobre este punto.

12 Por supuesto, ello no implica que abordar normatividad sea en manera alguna irrelevante. Remito a lo que he sostenido en Rapetti (2019, cap. 4), aunque volveré sobre esta cuestión infra sección V. 
Pablo A. Rapetti

portamiento habitual y el seguimiento de reglas [rule-following]. El hábito de obediencia es simplemente una regularidad de conducta, mientras que seguir una regla implica tomar las normas jurídicas como razones para hacer aquello que el derecho manda y como razones para criticar a aquellos que se aparten de las normas.

Bernal mantiene la objeción de Hart, como generalmente hace la teoría del derecho contemporánea, y la utiliza para apoyar la tesis de la práctica social. Pero, ¿es justa la objeción de Hart? Uno podría sostener que no. En primer lugar, Austin no reduce la normatividad del derecho a la amenaza de sanción. El hecho de que las normas sean generalmente guías para la acción depende de un complejo conjunto de hechos psicológicos, del tipo de los que investiga la filosofía moral. En segundo lugar, esta explicación sigue siendo compatible con la idea de la existencia de reglas como razones para la acción. Hechos tales como que cierta autoridad haya sido obedecida en el pasado, la preferencia por una cierta forma de gobierno político, o la preferencia por un sistema de gobierno en lugar de la anarquía, pueden ser entendidos como razones que condicionan la deliberación práctica de los destinatarios del derecho y explican por qué algunos miembros de la comunidad ven al derecho como un conjunto de reglas que regula adecuadamente las conductas. Para Austin esas razones son simplemente una parte de la explicación psicológica del comportamiento individual, que está fuera del ámbito de la teoría del derecho empírica (Canale, 2014, pp. 84-85; nota omitida).

Como espero que ahora se note, aquí Canale se refiere solo a normativi-

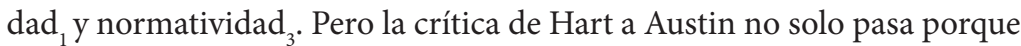
este último no dé cuenta de normatividad ${ }_{3}$, sino - y de manera más fundamental - porque la "teoría empírica" austiniana reduce explicativamente las relaciones normativas a términos fáctico-causales que oscurecen, más que aclarar, nuestra comprensión de los fenómenos jurídicos. Piénsese en términos de la distinción de Kelsen (1979, pp. 89-90) entre los principios de imputación y de causalidad. La crítica de Hart, según creo, tiene que ser entendida (en parte) como acusando a Austin de indebidamente fundir ambos. Canale apela a ciertos tipos de hechos nombrados por el propio Austin y afirma que pueden ser entendidos como (constituyendo) razones 
Ontología social y práctica jurídica: la actualización de un debate

que explicarían "por qué algunos miembros de la comunidad ven al derecho como un conjunto de reglas que regula adecuadamente las conductas". Pero la crítica de Hart es que la reducción factualista nos impide siquiera atribuirles a ciertos miembros el ver al derecho como un conjunto de reglas, sean adecuadas o no. ${ }^{13}$

Pero volvamos brevemente al "problema de la colectividad". Tanto Canale (2014, pp. 78-86) como Ekins (2014, pp. 119-124) discrepan de Bernal en cuanto a la posible lectura colectiva. Los dos sostienen que la lectura apropiada es la distributiva y para ello ofrecen varios elementos exegéticos. Canale es particularmente enfático, dado que no solo sostiene que la teoría de Austin sería una pobre encarnación de TPS, sino que se trataría, más bien, de una concepción fatalmente encaminada a rechazar TPS. Para Canale, la teoría de Austin presenta al derecho como una empresa primordialmente no cooperativa, basada en la relación vertical y de dominación entre soberano y súbditos. La cooperación puede tener lugar, pero no es condición necesaria para la existencia del derecho.

Bernal, en su réplica (2014b, pp. 159-161), trae a colación un pasaje de la obra de Austin en la que describe el paso de una "sociedad natural" a una "sociedad política”, jurídicamente organizada. El paso está dado justamente porque el grueso de la sociedad comience a obedecer habitualmente a alguien, erigiéndolo así en el soberano. Lo notable es que allí Austin alude a una organización temporaria de varias comunidades que voluntaria y conjuntamente se someten a efectos de repeler a un tercero, enemigo, extranjero (Austin, 1995, p. 177). Ello muestra que es posible que una "sociedad política" se forme mediante AICR.

En mi opinión, se trata de una réplica insuficiente a la observación de Canale, porque este admite que en la concepción austiniana puede haber cooperación, e incluso la formación de un derecho mediante cierta forma de cooperación. Su punto es que esto no es de ninguna manera necesario, y

${ }^{13}$ Esto explica - solo por mencionar un ejemplo acaso no tan obvio- las disquisiciones de Hart, al inicio de su presentación de la concepción de Austin, sobre por qué reconstruir dicha postura como parangonando las normas a órdenes respaldadas por amenazas, en lugar de a mandatos. El apunte es acaso no tan obvio, porque es usual referirse a la teoría de Austin como "del mandato" incluso cuando se la aprecia a partir del clásico análisis hartiano, dejando de lado las disquisiciones a que aquí aludo. Véase Hart (1963, pp. 23-26). 
ello parece fundamento bastante para sostener que, desde un punto de vista austiniano, el derecho no es fundamental o esencialmente una práctica social. Lo más interesante de apuntar, en todo caso, es que esta discusión nos da buenas razones para intentar introducir una distinción que en el debate bajo examen no se plantea. Uno podría distinguir entre (1) la verdad de TPS en relación con la génesis del derecho, de un ordenamiento jurídico, y (2) la verdad de TPS en relación con el funcionamiento y continuidad del derecho. Esto puede dar pie a una complejización del debate porque podría resultar el caso que

a) El derecho necesariamente se funde en una práctica social y necesariamente haya de funcionar como una.

b) El derecho no necesariamente se funde en una práctica social, pero necesariamente haya de funcionar como una.

c) El derecho necesariamente se funde en una práctica social, pero no necesariamente haya de funcionar como una.

d) El derecho no necesariamente se funde en una práctica social, ni necesariamente haya de funcionar como una. ${ }^{14}$

\section{Hart y TPS}

La teoría de Hart tiene un potencial aparentemente superior para presentar al derecho como conforme a TPS. Ello se debe al juego del concepto de aceptación. La aceptación permite el paso de los meros hábitos sociales a las reglas sociales. Cuando un hábito social, es decir, una conducta socialmente convergente, es considerada por parte de quienes convergen en la conducta en cuestión como una regla - es aceptada en tanto que regla (social)_, entonces se convierte efectivamente en regla (social). Que se la considere como una quiere decir que se asuma que la conducta convergente

${ }^{14}$ La distinción es ciertamente cercana a la que propone Tuzet entre ontología-proceso y ontología-resultado. Pero no se identifica con ella. Entre otras cosas, porque supone una consideración funcional y por ende diacrónica del devenir de los ordenamientos jurídicos. La primera distinción, en cambio, parece implicar una consideración de la ontología-resultado (derecho) solo desde un punto de vista sincrónico. 
es de algún modo debida, lo que se manifiesta (1) en el hecho de que se critiquen las desviaciones respecto de ella (y eventualmente se elogie el cumplimiento), dando lugar a una presión pro-conformidad, y en que, (2) de tener que justificar tales críticas, la justificación apele - al menos en parte- a la existencia de la regla al respecto. ${ }^{15}$

La presentación que Bernal hace de estas ideas (2014a, pp. 32-33) me resulta confusa. Sostiene que la consideración de que la conducta convergente es de algún modo debida constituye lo que Hart llama el "aspecto interno de las reglas" y tendría "una naturaleza diferente" de la de (1) y (2). A su vez, Bernal afirma que este elemento adicional

[s]e refiere a la actitud que los miembros de la sociedad tienen frente al derecho. Hart denomina esta actitud: el punto de vista interno. Como Shapiro ha aclarado, el punto de vista interno no se refiere simplemente al punto de vista de los miembros de la sociedad o de los participantes en la práctica jurídica social. El referente de este punto de vista es, más bien, un tipo especial de actitud que estos participantes pueden tener: la actitud de aceptación de las reglas. La actitud de aceptación de reglas es una disposición, en sentido filosófico, a lo que el aspecto interno de las reglas describe, a saber, al establecimiento de un patrón de comportamiento para que sirva como estándar de conducta, es decir, como una guía de conducta dirigida a todos... (Bernal Pulido, 2014a, p. 33; nota omitida, cursivas en el original)

Quizás sea demasiada la profusión de referencias: el aspecto interno de las reglas se refiere al punto de vista interno de las reglas, que a su turno se refiere a la actitud de aceptación, que es una disposición "al" aspecto interno de las reglas, que describe el establecimiento de un patrón de comportamiento para que sirva como estándar de conducta. Creo que todo esto tiene poco sentido.

He aquí un intento de pasar en limpio las ideas de Hart:

${ }^{15}$ Cfr. Hart (1963, pp. 129-130, 142-144, 250-251; 1982, p. 146; 1958, pp. 92-93). 
a) El aspecto interno de una regla está dado por el hecho de que se la acepte. Más precisamente, es la propiedad de ser aceptada, lo cual fija su contenido en tanto que regla.

b) Decir que alguien "acepta" una regla equivale a decir que "tiene el punto de vista interno" respecto de esa regla (o alguna expresión similar). ${ }^{16}$

c) Que se acepte una regla quiere decir que se la toma como parámetro para la guía y evaluación de las conductas que la regla cubra. ${ }^{17}$

d) Que así se la tome supone que se tiene la disposición de usarla como guía y estándar de evaluación.

e) La manifestación del uso ${ }^{18}$ está fundamentalmente dada por la calificación efectiva de conductas en función de la regla y por la crítica a las desviaciones y el elogio del cumplimiento. ${ }^{19}$

16 Así, el "aspecto interno" es propio de las reglas, es un componente de ellas, mientras que el "punto de vista interno" es propio de los agentes: acaso es un estado mental, acaso una disposición en sentido específicamente comportamental (me inclino por la primera opción, que a su vez puede comprender una traducción en términos disposicionales no-deterministas; pero este no es el contexto en el que detenerse en esto). Lo que vale la pena aclarar ahora es que (1) decir que el aspecto interno de una regla está dado por el hecho de que se la acepte equivale a decir que el aspecto interno es el hecho de que se tenga o profese respecto de ella el punto de vista interno; (2) la aceptación solo es directamente condición necesaria para la existencia de reglas sociales. Y por ende solo estas deben necesariamente contar con un "aspecto interno". Otras reglas, como las jurídicas, no necesariamente requieren ser aceptadas para existir. Al menos no directamente: la "existencia jurídica" (validez) de reglas requiere de RR, que es una regla socio-jurídica; ella sí tiene una existencia condicionada a la aceptación.

17 Las reglas no describen el establecimiento de un patrón de conducta para que sirva como guía; lo establecen. Ello mediante la descripción (o asunción/presuposición de una descripción) de una acción o estado de cosas al que se califica deónticamente (relación antecedente-consecuente). Como es obvio, aquí y en el texto principal solo me refiero a reglas primarias. La aceptación de reglas secundarias funciona de manera análoga, pero con particularidades sobre las cuales Hart es lagunoso. Para dos propuestas sobre cómo detallar la aceptación de reglas secundarias véase Kramer (2018, pp. 47-50).

${ }^{18}$ Lo que implica, desde luego, la manifestación de la disposición de uso.

19 Las "críticas", sin embargo, pueden limitarse al señalamiento de que una cierta acción es "incorrecta" en función de la regla en cuestión. Ello porque se puede aceptar la regla en tanto que social (o en tanto que jurídica, etc.) pero, a la vez, rechazársela desde otros puntos de vista (e.g., moral). Una acción puede ser tenida tanto por correcta como por incorrecta según distintos puntos de vista, es decir, según (la aceptación de) distintas reglas. Otro tanto, en signo opuesto, sucede con los "elogios". Las manifestaciones son por supuesto necesarias para que alguien pueda decir adecuadamente de otro que es un aceptante de la regla. 
Ontología social y práctica jurídica: la actualización de un debate

Con todo, me temo que la reconstrucción de Bernal presenta ulteriores problemas. Así, afirma lo siguiente:

Hart sugiere que la aceptación de reglas es una disposición (...) [E]sto puede entenderse como la actitud de la mayoría de los miembros de la sociedad de estar dispuesto a - o en tener la propensión de- asumir ciertos patrones de comportamiento consistentes en adoptar las normas jurídicas como guías de conducta futura, en la tendencia a actuar de acuerdo con dichas normas y a criticar toda desviación de ellas. De esta manera, estos patrones de comportamiento pueden servir como una razón para la acción conforme a las normas jurídicas y como una crítica para el comportamiento que de ellas se desvía. Sin embargo, esta versión del concepto de regla de aceptación [sic] parece muy débil, ya que admite una interpretación muy problemática de acuerdo con la cual lo único necesario es la actitud de los miembros de la sociedad en el sentido de que es posible que ellos asuman los patrones de comportamiento de derecho como guías de conducta, y no ya la actitud consistente en que ellos de hecho asumen dichos patrones como guías para su conducta futura. Esto no parece consistente con el análisis de Hart acerca del aspecto interno de las reglas, en el cual, es evidente que lo que se exige es que los miembros de la sociedad de hecho consideren los patrones de comportamiento establecidos por el derecho como estándares generales para ser seguidos por el pleno de la sociedad (Bernal Pulido, 2014a, pp. 34-35; nota omitida, cursivas en el original).

Para empezar, hay un inconveniente de circularidad al explicar la disposición como disposición. Pero la cuestión más relevante es que en este pasaje se evidencia una confusión entre tomar una regla como guía y actuar de conformidad con ella. Uno puede tomar efectivamente como guía para su conducta una cierta regla y esto no se desmiente por el hecho de que en ocasiones no actúe de conformidad con ella. Por supuesto, el apartamiento no puede ser completo, sistemático, ni mayoritario. Por otro lado - y de manera más fundamental—, debe recordarse algo señalado poco más arriba: ser aceptante de una regla solo requiere que se la use en el sentido de parámetro calificador de conductas. Esto ciertamente supone 
una disposición a (inclinación hacia) la realización de acciones concretas, ${ }^{20}$ pero no tiene de ninguna manera por qué resultar determinante de acciones concretas o, dicho de otro modo, no tiene por qué implicar que la regla es (en efecto) proveedora de razones concluyentes para la acción. Ni que se considere que las provee. Lo débilmente "posible" aquí no es que los ciudadanos asuman los patrones de comportamiento de derecho como guías de conducta, sino que actúen de conformidad con los patrones asumidos como guías, precisamente porque el asumir algo como guía no implica que necesariamente se lo acatará.

Finalmente, Bernal trae a colación la interpretación de Shapiro según la cual la aceptación implicaría un compromiso de actuar conforme a la regla, y sostiene que se trata de una visión demasiado intelectualizadora, porque "atribuye a los agentes una consciencia interna con relación a las reglas que va más allá de la que en realidad puede existir” (Bernal Pulido, 2014a, p. 35). En cambio, afirma, puede haber aceptación irreflexiva producto del "entrenamiento" que genera la presión social sostenida, sin representaciones ni intenciones de por medio. ${ }^{21}$

Tanto Canale como Ekins discrepan de esto. El primero sostiene que hacer inintencionadamente lo que una regla establece no supone aceptación alguna, sino solo actuar de acuerdo con la regla (Canale, 2014, p. 89). Ekins, por su parte, afirma que

[s]i Shapiro intelectualiza demasiado, Bernal hace desaparecer toda intelectualización (...) Se puede tener una intención condicional y actuar de conformidad sin mucha auto-conciencia. Pero aun así se debe adoptar algún curso de acción, eligiendo un fin y sus medios: específicamente, uno elige hacer X cuando o si Y sucede. Las "presiones sociales... que equivalen a una forma de entrenamiento" solo

${ }^{20}$ La aceptación no es una actitud cognitiva, sino conativa. Véase (Caracciolo, 1988, p. 50); y (Toh, 2005, pp. 79 y 89).

${ }^{21}$ Según Bernal, las reglas aceptadas de ese modo constituirían parte del "trasfondo de intencionalidad" de que se nos habla en Searle, 1992 (cap. 5). Esto parece incorrecto, dado que Searle define dicho trasfondo como un conjunto de capacidades mentales no representacionales (p. 152). Difícilmente ello abarque reglas. Sostener tal cosa sería caer en un error categorial análogo al que veremos más abajo. Más bien, parece que esas capacidades del trasfondo son las que nos llevarían a aceptar ciertas reglas en la manera que propone Bernal. 
explican la aceptación de reglas, digo, si llevan a las personas a adoptar la intención relevante (condicional), mostrándoles que así es como se debe actuar. Las presiones sirven para provocar una disposición continua si alientan al agente a responder a las condiciones relevantes actuando según la regla que ya adoptó (eligió) (Ekins, 2014, p. 125).

He de discrepar de los tres nuevamente. Hart admite que la aceptación puede ser irreflexiva, y en esta medida Bernal lleva razón. Pero, en cambio, Bernal des-intelectualiza efectivamente demasiado al querer amoldar el desarrollo de la aceptación de reglas al mero entrenamiento tácito producto de presiones sociales. Si bien es cierto que se puede actuar qua aceptante incluso cuando se cumple con una regla de modo inintencionado, este solo será el caso si para esa situación resulta verdadero el contrafáctico " $\mathrm{Si}$ le hubiesen pedido justificación de su acción, habría aludido a la regla (o aludirá a ella si se la piden)". El momento de la acción puede ser irreflexivo, pero no el eventual momento de la justificación. Esto, sin embargo, no es tan exigente (o intelectualizador) como para implicar que uno acepte reglas solo si las elige y se traza un plan de acción a futuro en relación con ellas.

En todo caso, Bernal concluye que la teoría de Hart, en definitiva, es poco amigable con TPS, porque el concepto de aceptación también admitiría tanto una lectura colectiva como una distributiva: cada quien puede ser aceptante por puras razones personales (2014a, pp. 36-37). Además, es sabido que — según Hart - para la existencia de un (caso paradigmático de) ordenamiento jurídico en una comunidad, basta con que la generalidad de la población obedezca las reglas, actúe de conformidad con ellas; la aceptación no se exige más que al grueso de los funcionarios (en particular, jueces). Esto haría del derecho no una práctica social, sino una práctica oficial (Bernal Pulido, 2014a, p. 39). Y ello traería ulteriores inconvenientes para la teoría de Hart. Primero, no podría explicar por qué la práctica oficial da lugar a reglas que son vinculantes. Ni respecto de los funcionarios ni - mucho menos - respecto del resto de los ciudadanos. "En segundo lugar", según Bernal, la teoría de Hart no podría explicar "la normatividad del derecho". Con este uso de la palabra hace referencia a la fuente y/o carácter de la vinculatoriedad del derecho: bajo esta rúbrica Bernal sigue la reconstrucción que de Hart hace Shapiro, según la cual las reglas jurídicas "producen" normatividad de tipo jurídico, pero no moral, 
Pablo A. Rapetti

y lo que quiere decir el concepto de normatividad es que las autoridades oficiales orientan su punto de vista interno hacia las reglas jurídicas. Esto implica que ellos hacen juicios normativos de acuerdo con los cuales adoptan las reglas jurídicas como estándares para guiar y evaluar la conducta. Esto se lleva a cabo mediante juicios normativos sobre la validez jurídica. Hart los denomina enunciados internos. Los enunciados internos son enunciados fácticos, en el sentido de que solo expresan la existencia del compromiso de tomar a las reglas como un estándar para las conductas futuras. De esta manera, la regla de reconocimiento, como una práctica social es un hecho que da lugar a otros hechos, señaladamente, las acciones colectivas intencionales recurrentes, llevadas a cabo por las autoridades oficiales que juzgan los casos sobre la base de las reglas jurídicas, y la aceptación interna de esas reglas como guías para la conducta futura. En consecuencia, en la teoría de Hart un "ser" deriva de un "ser". Esto respeta el principio de Hume y no incurre en una falacia naturalística (Bernal Pulido, 2014a, p. 42). ${ }^{22}$

Según Shapiro, sin embargo, el problema estribaría en que reducir RR y otras reglas sociales a prácticas sociales supone cometer un error categorial, porque reglas y prácticas pertenecen a categorías metafísicas distintas:

[1]as reglas son entidades abstractas. Son como los juegos, los números, las historias, las proposiciones y los conceptos — son entidades del pensamiento, no objetos que existen en el espacio y el tiempo(...) Las prácticas, por otra parte, son eventos concretos. Tienen lugar en el mundo natural e interactúan causalmente con otros eventos físicos (Shapiro, 2014, pp. 141-142; cursivas en el original).

En este punto Bernal se aparta de Shapiro, sosteniendo que una interpretación adecuada no endilga a Hart este error categorial, porque Hart sostenía más bien que las prácticas sociales fundan reglas sociales. En este sentido, RR es el fundamento del sistema jurídico (Bernal Pulido, 2014a, p. 43). Y RR estaría fundada en una práctica social. Como se ve, aquí Bernal

22 Me temo que calificar a los enunciados internos de fácticos es un craso error, además de contradictorio con justo antes haberlos catalogado como normativos. Toh, a quien se alude en ese pasaje, no comete ese error. 
acude a la distinción entre reducción y fundamentación que he mencionado en la sección II.

No obstante, Bernal mantiene que Hart no consigue demostrar que las prácticas sociales puedan realmente "generar" (fundar) reglas sociales. En particular, no lograría explicar la vinculatoriedad de RR y de las demás reglas creadas de acuerdo con ella y cómo imponen deberes. Nótese que esto muestra que para Bernal (1) "el problema de la normatividad del derecho" no es en realidad uno "segundo", distinto del de la vinculatoriedad, y que (2) demostrar que las prácticas sociales pueden realmente fundar reglas sociales de algún modo equivaldría a -o implicaría - demostrar dicha vinculatoriedad (o sea, que fundamentación metafísica y fundamentación normativa - en sentido robusto- equivaldrían o estarían conectadas por implicación).

Finalmente, Hart tampoco podría explicar por qué las autoridades son autoridades, según el clásico argumento de la circularidad: RR, qua regla última, parece tener que ser la fuente última de la autoridad jurídica, pero su existencia depende de la práctica de las autoridades (Bernal Pulido, 2014a, pp. 44-45). Estas dificultades mostrarían que, en definitiva, la teoría de Hart es incompatible con TPS.

La discusión acerca de la vinculatoriedad se trata de lo que más arriba he denominado "normatividad" y ya la hemos repasado. Pasemos entonces a la relación entre reglas y prácticas. Canale sostiene (2014, p. 88) que la conexión es "interna" - explicativa-, aunque está de acuerdo con que corresponden a dos categorías metafísicas diferentes. ${ }^{23}$ El concepto de regla social explica lo que una práctica social es. De hecho, una práctica social no podría ser explicada independientemente de la referencia a reglas sociales y viceversa. Esta misma conexión explicativa permitiría rechazar la objeción relativa a la incapacidad de dar cuenta de la noción de autoridad, en la medida en que RR sea considerada como "definicional". Y cita a Marmor sosteniendo que las reglas que constituyen un cierto tipo de actividad pueden también constituir los roles que las personas ocupan en ella (Canale,

${ }^{23}$ Curiosamente, Canale atribuye a Bernal el rechazo de la tesis de que reglas y prácticas correspondan a categorías distintas. Se trata, evidentemente, de un desliz. 
Pablo A. Rapetti

2014, pp. 91-92). ${ }^{24}$ Sumándole algo de detalle al punto, puede decirse que RR se forma mediante la actividad de ciertos agentes que luego la propia $\mathrm{RR}$, ya constituida, erige en autoridades oficiales. ${ }^{25}$

Me parece que esto es plausible como parte de la respuesta a la objeción. Sin embargo, debe complementársela; porque el punto central, a mi entender, lo ofrece el propio Hart al decir que RR puede ser considerada tanto desde el punto de vista interno como del externo. Y ello implica que en esta discusión hay que trazar además una distinción entre cuestiones lógicas, por un lado, y cronológicas, por el otro. Quienes con su actividad dan origen a RR, han de (o, al menos, pueden) ser considerados ex post como autoridades, aunque obviamente no hayan podido ser considerados como tales durante la realización de dicha actividad generadora. Razones de espacio me impiden desarrollar en extenso esta idea, pero como dije, me parece que está ya implícita en el trabajo de Hart. ${ }^{26}$

Asimismo, esta objeción exige volver sobre la clásica discusión sobre si $R R$ es efectivamente definicional o, en cambio, una regla de deber. No es un dato menor que casi todos los iusfilósofos anglosajones consideran que es una regla de deber, en ello aparentemente contra Canale/Marmor. En el medio hispanohablante, sin embargo, la concepción definicional tiene algo más de predicamento, a partir del seminal debate entre Eugenio Bulygin y Juan Ruiz Manero, terciado por Ricardo Caracciolo. ${ }^{27}$ Mi posición es que se trata de una falsa disyuntiva: creo que la caracterización hartiana de RR encaja muy bien con la conocida concepción de Von Wright sobre las reglas determinativas (o, quizás aún mejor: con la que ofrece sobre las normas de costumbre). Ahora bien, una parte acaso descuidada de esa caracterización es que tales reglas pueden ser apreciadas desde dos puntos de vista: desde el de la actividad que determinan y desde el de los actores involucrados en ella. Desde el de la actividad las reglas son, en efecto, constitutivas; pero desde el de los actores son reglas (entre otras no determinativas, esto es, prescripciones) que establecen qué "movidas" dentro de la actividad tienen

${ }^{24}$ Véase también Marmor (2010, p. 56).

${ }^{25}$ Por lo demás, Canale asiente en que la teoría de Hart no casa bien con TPS, pero afirma también que ello no configura necesariamente un defecto (2014, p. 92).

26 Véase Hart (1963, pp. 138-139).

27 En el n 9 de la revista Doxa (1991). 
Ontología social y práctica jurídica: la actualización de un debate

permitidas y qué otras no (Von Wright, 1970, p. 26-29). Así concebida, RR es definicional $y$, a la vez, de deber. ${ }^{28}$

Ekins, por su parte, afirma que la lectura distributiva de la aceptación hartiana no es posible. Ello por el análisis que ofrece Hart de las reglas sociales, y el hecho de que estas requieren presión colectiva en favor de su cumplimiento. Finalmente, porque las reglas jurídicas secundarias, en el contexto del conocido argumento cuasigenealógico de Hart, ${ }^{29}$ parecerían, en general, producto de la acción colectiva. Respecto de RR en particular, Ekins subraya que "el punto de la regla es proveer una regla para el grupo", lo que también contribuiría a la lectura colectiva. Asimismo, dice que la aceptación por parte de la generalidad ciudadana no es estrictamente necesaria pero tampoco es irrelevante, con lo que - en todo caso- el derecho a veces resultará una práctica oficial, pero a veces una genuinamente social (Ekins, 2014, pp. 128-129). Esta réplica parece desencaminada: la discusión está más bien trabada en términos de si el derecho es necesariamente una práctica social. Por lo demás, Ekins parece querer extraer demasiado del argumento cuasigenealógico, que solo apela a lo conceptualmente indicativo de una hipótesis o ficción, y no pretende mostrar que las reglas secundarias surjan, históricamente, de un determinado modo en especial.

En cuanto a que el punto de RR sea proveer de criterios para el grupo (de esto se trata, en propiedad), Bernal responde (2014b, p. 165) que ello todavía no define si la contribución de los individuos cuya actividad da lugar a RR es una que ha de ser realizada mediando intenciones en primera persona singular o mediando intenciones comunes. Esto es, el punto no alcanzaría para hacer necesaria una lectura colectiva, porque seguiría siendo compatible con una distributiva. No me ocuparé del detalle de esta respuesta. ${ }^{30}$ En cambio, me interesa señalar que — sorprendentemente-

${ }^{28}$ Además, todo esto es asumiendo que se trata de una regla única. Quizás ello constituya una ficción, y RR sea más bien un complejo de reglas varias, algunas definicionales y otras de deber.

${ }^{29}$ Es decir, el argumento relativo al paso de una hipotética sociedad "pre-jurídica", a una jurídicamente organizada.

${ }^{30}$ Que me parece poco convincente. Creo que ni el ejemplo sobre la evacuación del teatro, ni el de la regla sobre la sana distancia con interlocutores, satisfacen el cometido por el que Bernal los propone. Pero no podría explayarme sobre esto aquí. 
ninguno de los textos bajo análisis hace referencia a que Hart explícitamente señala que $R R$ requiere de intenciones comunes. En su post scriptum a El concepto de derecho, el autor inglés atiende a una conocida observación que le formuló Ronald Dworkin, tomándose de la distinción trazada por este último entre consensos por convicción y por convención. Que se llegue a un consenso por una u otra, equivale, respectivamente, a que se lo obtenga mediante acciones signadas por intenciones en primera persona singular o a intenciones comunes. Habiendo suscripto la distinción, Hart afirma (2000, p. 33) que las reglas sociales en general, y RR en particular, deben ser entendidas como constituidas mediante consensos por convención.

Ahora bien, esta clarificación no basta para asegurar el encaje perfecto de la teoría de Hart con TPS. Porque, a pesar de que la conformación de RR requiera de intenciones comunes, los sujetos relevantes son los jueces, que siguen siendo un pequeño subgrupo dentro de la comunidad. En principio, entonces, parece que Bernal prevalece en el examen de fondo: el derecho, desde una perspectiva hartiana, no necesariamente es (se funda en) una práctica social, sino oficial. Pero llegados a este punto podemos pretender un nuevo refinamiento de la discusión. El criterio de Bernal para considerar "social" a una práctica parece exigir implícitamente que debe haber AICR de parte de todos o una gran mayoría de los miembros de la sociedad en cuestión. Así, el derecho de una sociedad determinada sería una práctica solo oficial si se aplica básicamente a todos sus miembros a pesar de existir (y/o depender en su continuidad) solo en virtud de las acciones e intenciones de un subgrupo dentro de ella. Sin embargo, me parece que este criterio eminentemente "mayoritarista" no es tan obvio ni se sigue ineludiblemente de las definiciones de partida. En particular, uno puede preguntarse si el micro-debate apuntado más arriba sobre la agencia grupal y la representación no ofrece motivos para relajar este mayoritarismo. Como vimos, el propio Bernal sostiene que su concepción de la acción colectiva admite la representación, de modo que las acciones (e intenciones) de unos pocos cuenten como del grupo en su totalidad. Uno podría preguntarse por la aplicabilidad de esa idea a la actividad judicial tal como es requerida para la conformación de RR y la consecuente fundación de un orden jurídico en la teoría de Hart. En otras palabras: la estrategia de Bernal para admitir la representación en la acción colectiva podría quizás servir, asimismo, para 
Ontología social y práctica jurídica: la actualización de un debate

reconstruir la teoría hartiana como representando al derecho qua práctica social, compatibilizándola así, del todo, con TPS.

\section{Normatividad y constitución metafísica}

Hay otros dos aspectos de potencial sofisticación del debate que me gustaría introducir para finalizar este balance. El primero tiene que ver con la asunción, propia del planteo originario de Bernal, de que las cuestiones de normatividad 1 hacen parte del debate. En este sentido, una teoría del derecho que encaje adecuadamente con TPS sería una que logre, entre otras cosas, explicar (justificar) la obligatoriedad del derecho, tanto respecto de los funcionarios como del resto de los ciudadanos. Esto no es en absoluto obvio. Por lo pronto, una enorme parte de la metaética analítica contemporánea parece militar en contra de una asunción análoga relativa al dominio moral y podríamos intentar trasladar esas consideraciones al campo jurídico.

Como es sabido, la metaética es una disciplina filosófica consistente en un discurso de segundo orden ocupado de las cuestiones metafísicas, conceptuales y semánticas que subyacen al discurso ético o moral de primer orden. ${ }^{31}$ Muchos autores dedicados a la metaética sostienen que la elucidación de, e.g., la cuestión metafísica (y, en particular, ontológica) de si existen o no hechos morales es neutral desde el punto de vista moral. Es decir que, cuando se afirma que existen los hechos morales X y Z, no se está diciendo ni implicando nada acerca del estatus moral de acciones o estados de cosas determinados. Esto es lo que permite concebir la metaética como una disciplina explicativa pero no justificativa, genuinamente de segundo orden, avalorativa. Esta caracterización es defendida por metaéticos de las más diversas posiciones. ${ }^{32}$ Tal como puede sostenerse esto en punto a la relación ética-metaética, puede sostenérselo para la relación derecho-teoría del derecho: no es claro que la

${ }^{31} \mathrm{Al}$ menos en una concepción "estrecha". En una algo más amplia se incluyen también cuestiones psicológicas y epistemológicas. Véase Schroeder (2008, p. 8).

${ }^{32}$ Curiosamente, uno de los filósofos más críticos de la supuesta neutralidad metaética "proviene" de la discusión iusteórica: véase Dworkin (1996; y 2011, parte 1). Para algunas defensas de la concepción estándar de la metaética, véase Blackburn (1993, cap. 8); Bloomfield (2009); Ehremberg (2008); Rapetti (2019, pp. 242-253). 
elucidación de la cuestión ontológica de si el derecho es una práctica social deba incluir una "elucidación" (justificación) de normatividad. Hay buenas razones para separar, al menos en principio, las cuestiones ontológicas de las normativas ${ }^{33}$. Esto es lo que Austin y Hart sostenían.

El segundo aspecto de potencial sofisticación tiene que ver con el modo en que concebir que el derecho esté fundado en una práctica social. El debate en análisis no especifica cómo concebir dicha fundación o constitución, aunque, como hemos visto en el repaso sobre RR, Bernal tiene presente la distinción entre la reducción de esta a una práctica social y el que RR esté fundada en una práctica social. Propongo, a modo ejemplificativo, que tengamos en cuenta dos herramientas filosóficas con las que posiblemente abordar este asunto.

La primera es el concepto de fundamentación [grounding], de discusión bastante reciente en la metafísica analítica. El concepto se propone para

${ }^{33}$ Una alternativa consiste en sostener que la separación entre cuestiones ontológicas y normativas es perfectamente posible, pero por una razón diferente; a saber, que la elucidación de normatividad ${ }_{1}$ es absolutamente posible sin que ello implique ninguna forma de justificación. El procedimiento intelectual requerido sería conceptual/descriptivo, tal como sucede con los otros tipos de normatividad. Esto mismo se me sugiere en una revisión anónima para Discusiones, en la que se cita a Raz (1999) como un ejemplo de trabajo en que se ofrece una elucidación tal —esto es, sin asumir posiciones valorativas sustanciales- de normatividad $_{1}$. Me inclino a pensar que esta no es una alternativa viable. Una cuidadosa discusión del punto es imposible aquí, pero mínimamente podría decirse al respecto lo siguiente: (1) es difícil ver cómo puede desentrañarse el contenido de nociones normativas con una mínima pretensión de completitud sin embarcarse en el razonamiento normativo, al menos mientras se distinga entre lo que se considera el contenido de una determinada noción tal y el ("verdadero" o "correcto") contenido de la misma; (2) si la distinción mencionada no se sostuviese, en cambio, perdería razón de ser la distinción misma entre elucidación y justificación que supuestamente nutre a la alternativa bajo análisis, tanto como a la razón que yo he ofrecido en el cuerpo de texto. Además, (3) he definido normatividad como la cuestión de si el derecho ofrece razones concluyentes para la acción: me parece que el carácter de concluyente o no de una razón cualquiera es, indefectiblemente, materia de discusión sustancial. En cuanto al ejemplo con la obra de Raz, yo diría (4) que en general se trata, en lo que nos compete, de una elucidación de normatividad ${ }_{2}$ y no de normatividad. De ahí, por ejemplo, que Raz caracterice al derecho como pretendiendo supremacía, pero no como supremo. También podría agregarse (5) que en los pasajes en que Raz en efecto se ocupa allí de normatividad, lo hace asumiendo posiciones sustanciales. Pero todo esto nos llevaría demasiado lejos. En todo caso, lo que he intentado en el cuerpo de texto ha sido mostrar al menos una razón por la que deslindar la elucidación ontológica de la normativa. 
Ontología social y práctica jurídica: la actualización de un debate

caracterizar con algo más de detalle la idea de dependencia metafísica, que durante mucho tiempo ha sido empleada como primitiva, i.e., solo intuitiva y no-analizable. ${ }^{34}$ Dos o más hechos ${ }^{35}$ pueden estar ligados entre sí mediante una relación de fundamentación y esto es lo que quiere decir que un hecho esté constituido por, se dé en virtud de o por (etc.) otro(s) hecho(s). ${ }^{36}$ Como un hecho puede estar fundamentado por otros varios, la relación de fundamentación puede ser parcial, y no necesariamente total.

Así, el hecho de que

(a) Juan Carlos, Silvia... y Mercedes caminan por la calle Alsina,

fundamenta el hecho de que

(b) La masa de gente camina por la calle Alsina.

A su turno, (a) encuentra fundamentación en que

(c) Juan Carlos camina por la calle Alsina, (d), Silvia camina... etc.

Así, (a) fundamenta (b) de manera total, mientras que, e. g., (c), solo fundamenta parcialmente (a).

La relación parece estrictamente ontológica y no causal: (a) es la razón metafísica, la razón (no la causa) de la existencia de (b). ${ }^{37}$ La noción, asi-

34 Véase (Rosen, 2010, pp. 109-110). Sin embargo, esto implica que el concepto mismo de fundamentación termina siendo inanalizable, salvando las propiedades que usualmente se le atribuyen, tal como expondré a continuación en el cuerpo de texto. En contra: (Bricker, 2006). Por otra parte, muchos pretenden que la fundamentación es solo una forma de dependencia metafísica, entre otras.

${ }^{35}$ Hay quienes sostienen que, en propiedad, la relación es entre proposiciones, e. g., Bricker (2006, p. 254). Otros sostienen que es neutral a las categorías, de modo que puede conectar dos o más relata pertenecientes a distintas categorías ontológicas. Así, (Schaffer, 2009, pp. 355-356).

${ }^{36}$ En general se asume que esta manera de formular la idea presupone la existencia, en sentido propiamente ontológico, de relaciones. Kit Fine, uno de los grandes proponentes del concepto de fundamentación, prefiere no asumir ese compromiso y presenta la noción como una conectiva oracional no veritativo-funcional: (Fine, 2012).

${ }^{37}$ La relación tampoco es (puramente) modal, como sucede con el concepto de superveniencia: la superveniencia es una relación modal, esencialmente de covarianza, tal que si el hecho A (o acaso: las/algunas propiedades del hecho A) varía(n), también varía(n) el 
mismo, expresa la cuestión de la fundamentalidad señalada más arriba: (a) es un hecho metafísicamente más fundamental que (b). Otras características - formales - de la relación de fundamentación, son las que siguen: ${ }^{38}$

Irreflexividad: nada se fundamenta a sí mismo (así como ningún hecho puede dar lugar a sí mismo).

Asimetría: si (a) fundamenta (b), (b) no puede ser, por su parte, fundamento de (a).

Transitividad: si (a) fundamenta (b), y (c) $+(\mathrm{d})+\ldots$ fundamentan (a), entonces $(c)+(d)+\ldots$ fundamentan $(b)$.

Finalmente, y en la medida en que mostrar una relación de fundamentación entre dos hechos permita ofrecer una explicación (ontológica) del hecho fundamentado, también parece estar presente la característica - común a todas las relaciones explicativas- de la

No-monotonicidad: del hecho de que (a) fundamente (b) no se sigue que $(\mathrm{a})+(\mathrm{z})$ - donde $(\mathrm{z})$ es cualquier otro hecho arbitrario - fundamente (b). ${ }^{39}$

Un desarrollo de las investigaciones acerca del derecho en relación con TPS podría, entonces, tomar la forma de un estudio sobre si el derecho y los diversos elementos que lo componen se encuentran en relaciones de fundamentación con ciertos hechos sociales, caracterizadas como brevemente hemos repasado. Tomando la propuesta de Bernal como punto de partida, una pregunta a responder sería, por ejemplo, si una cierta norma jurídica (o el hecho de que exista) está en una relación semejante con determinadas AICR. Sin embargo, la introducción del concepto de fundamentación

hecho B (las/algunas propiedades del hecho B), lo que supone que B superviene "de", "en" o "sobre" A. Algunos autores afirman que toda relación de fundamentación implica también una de superveniencia, pero la conversa no parece de ninguna manera necesaria. Cabe señalar que, en un punto de la discusión bajo análisis, Bernal parece atribuirle a la noción de superveniencia un import metafísico del que carece: (Bernal Pulido, 2014b, p. 152).

38 Véase (Clark \& Liggins, 2012).

39 Sobre esto véase Rosen (2010, pp. 116-117). 
podría quizás llevarnos también a replantear si (o si solamente) las AICR serían fundamentos apropiados del hecho de que el derecho sea una práctica social en algún sentido interesante. ${ }^{40}$

Semejante intento es hecho por Brian Epstein (2015), quien concluye que la noción de fundamentación resulta útil pero insuficiente para dar cuenta de la ontología propia de las prácticas sociales. Por ello mismo introduce la segunda herramienta a la que pretendo referirme: el concepto de anclaje [anchoring]. Su trabajo ofrece el atractivo adicional de ocuparse específicamente del derecho, a modo ejemplificativo.

Una tarea esencial de la ontología social es la de establecer las condiciones de existencia de ciertos hechos sociales. Aplicada al derecho, esta tarea se traduce en la búsqueda de condiciones de existencia social de ciertos hechos jurídicos. Es un hecho (social y) jurídico, por ejemplo, que el papel que tengo en el bolsillo - llamémoslo " $\$$ " - es (cuenta como) un billete de $\$ 100$. La cuestión pasa por ver si ese hecho se encuentra fundamentado por otros y, eventualmente, cómo. ${ }^{41}$ Los fenómenos sociales - y el derecho en particular- se distinguen entre otras cosas por el rol que en esto cumplen las reglas. Es bien conocido el análisis que ofrece John Searle: el hecho de que \$ sea un billete encuentra fundamentación en el hecho - llámese "H\$\$” - de que fue emitido por la Casa de la Moneda, allí donde existe la creencia compartida, a nivel social, de que solamente son genuinos billetes los que emite la Casa de la Moneda. Podemos decir, entonces, que el hecho de que \$ sea un billete se basa en esa creencia generalizada. Pero la relación entre ambos hechos está mediada por una regla, que Searle denomina "constitutiva" y que es a su turno producto de la creencia generalizada. La forma general de las reglas constitutivas según Searle es:

$$
\mathrm{RC}=X \text { cuenta como } Y \text { en } C^{42}
$$

${ }^{40}$ Para una crítica general sobre la caracterización y utilidad del concepto de fundamentación, véase (Wilson, 2014). Para aplicaciones del concepto al análisis de la ontología jurídica, véase Chilovi \& Pavlakos (2019); y Gizbert-Studnicki (2016).

${ }^{41} \mathrm{O}$, mejor, se trata de investigar la clase de hechos involucrados: lo que haga que ciertos papeles sean billetes.

42 Donde “C” está por un contexto determinado: (Searle, 2010, p. 10). 
Epstein (2015, p. 77) sostiene que entre ambos hechos hay una relación de fundamentación y denomina "principios enmarcadores" [framing principles] a los elementos mediadores (que, afirma, pueden ser más variados que lo que captura la fórmula canónica searliana de RC). Estos principios dan las condiciones de fundamentación para la clase de hecho investigada; en nuestro ejemplo, que $\$$ es un billete. Los principios nos dicen qué fundamenta qué y pueden aplicarse tanto a hechos y situaciones actuales como potenciales. Son "enmarcadores" porque conforman un "marco" [frame], esto es, un conjunto de mundos posibles en los que las condiciones de fundamentación para hechos sociales están fijadas de una manera en particular. Así, infinitos hechos potenciales distintos, de una misma clase, se verán fundamentados de acuerdo con las mismas condiciones.

Aquí entra en juego el concepto de anclaje. Mientras que el concepto de fundamentación captura una relación entre dos o más hechos, el de anclaje captura la relación que hay entre los principios enmarcadores y los hechos que los establecen. ${ }^{43}$ En nuestro ejemplo, entre la regla -llámese " $\mathrm{R}_{\mathrm{s}}$ ”- según la cual ciertos papeles cuentan como billetes (e.g.: "Los papeles emitidos del modo $\mathrm{Z}$ por la Casa de la Moneda cuentan como billetes en nuestro país") y los hechos - llámense " $\mathrm{H}_{\mathrm{SR}}$ " - que la establecen (e.g., que de consuno admitimos que la Casa de la Moneda es la institución exclusivamente facultada para emitir billetes). ${ }^{44}$ De esta manera, el hecho de que \$ es un billete está fundamentado por $\mathrm{H} \$ \$$, dadas las condiciones que establece $\mathrm{R}_{\$}$, que por su parte existe en virtud de su anclaje en (o por) $\mathrm{H}_{\$ \mathrm{R}}$.

${ }^{43}$ Se ha objetado que esta es una razón insuficiente para postular la existencia de un tipo de relación distinto a la de fundamentación y, por ende, el concepto mismo de anclaje: véase (Schaffer, 2019) y una respuesta en (Epstein, 2019). La respuesta enfatiza (pp. 771-772) un rasgo del concepto de anclaje que no he referido en el texto principal, a saber, la tesis de la universalidad: las relaciones de anclaje funcionan de un modo universal. Esto quiere decir que, dado un hecho social de la forma $\mathbf{x}$ es $\mathbf{K}$ cuyas condiciones de fundamentación están ancladas por los hechos actuales (dados) $\mathbf{A}_{1} \ldots \mathbf{A}_{\mathbf{n}}$, el hecho $\mathbf{x}$ es $\mathbf{K}$ puede darse en otros mundos en los que $\mathbf{A}_{1} \ldots \mathbf{A}_{\mathbf{n}}$ no se den. Esto hace de las anclas una "herramienta universal" que puede instanciarse a través de diversos contextos de posibilidad de realización. En cambio, no puede haber fundamentación de un hecho en un mundo en el que no se dan también los hechos fundamentadores. De manera que entre ambos conceptos hay una importante diferencia modal. 
De manera sugerente, Epstein busca (2015, cap. 7) ilustrar el funcionamiento de su modelo metaontológico con la teoría jurídica de Hart. Considérese el siguiente hecho (jurídico):

Rafael V. es un homicida

Este hecho se fundamenta, asumamos, en el hecho

Rafael V. mató a Jorge B.

Entre ambos hechos, el principio enmarcador que da lugar a la fundamentación puede ser presentado como algo así:

\section{$\mathrm{PE}_{\mathrm{Rh}}=$ Para todo $\mathrm{x}$, si $\mathrm{x}$ mata a otro, $\mathrm{x}$ es un homicida}

$\mathrm{Y}$ a su vez podemos asumir que la formulación de $\mathrm{PE}_{\mathrm{Rh}}$ se infiere de lo que dice, por caso, el art. 79 del Código Penal argentino, ${ }^{45}$ que es el que expresa la norma que establece el delito de homicidio (en Argentina). Nótese que, de esta manera, el principio enmarcador de que Rafael V. es un homicida sería, en la terminología de Hart, una norma primaria. Como es sabido, según la teoría de Hart la existencia y validez de una norma como la expresada por el art. $79 \mathrm{CP}$ se puede deber a una compleja mezcla de hechos y normas: ${ }^{46}$ el hecho de que el Congreso la votó, el Ejecutivo la promulgó, diversos jueces en diversas ocasiones la han identificado y aplicado, etc., mediando normas secundarias de cambio y adjudicación. Como también es sabido, en última instancia la norma se debe a RR. Es decir que $\mathrm{RR}$ daría las condiciones generales de anclaje para $\mathrm{PE}_{\mathrm{Rh}} \mathrm{y}$ los hechos y normas que dieron lugar a la norma del art. $79 \mathrm{CP}$ serían particulares instanciaciones (del cumplimiento) de tales condiciones. A su turno, RR estaría anclada en hechos: los varios hechos de identificación y aplicación del derecho que hacen al regular uso de RR en un cierto contexto judicial,

45 Con más las normas sobre el ámbito de vigencia y aplicación de las demás normas, acaso algunos antecedentes interpretativos, etc.

${ }^{46} \mathrm{O}$ quizás: mezcla de hechos entre los que se cuentan hechos de la existencia de normas. 
la aceptación judicial de RR (manifestada en ese uso de RR, en los hechos de crítica a las desviaciones - es decir, al no uso de RR-, etc.).

Finalmente, cabe apuntar que una consecuente manera de comprender los perennes debates entre positivistas y antipositivistas sería como discusiones acerca de las anclas últimas de las normas jurídicas. Básicamente: mientras los positivistas sostienen que dichas anclas solo pueden consistir en hechos sociales, los antipositivistas sostienen que también son anclas, necesariamente, los/ciertos hechos morales. ${ }^{47}$

De acuerdo con lo dicho hasta aquí en esta última sección, es posible que - como se me sugiere en una lúcida revisión para Discusiones - valga la pena comprender el núcleo del debate Bernal-Canale-Ekins como acerca de cuáles son las anclas fundamentales del derecho. Creo que esto es ciertamente posible, aunque no obvio. Por lo pronto, la discusión no incluye la distinción fundamentación/anclaje y no estoy seguro de que aporte elementos para que la reconstruyamos en ella. Me parece más bien que tenemos que añadírsela. Otra fuente de dudas tiene que ver con la ya mentada distinción ontología-proceso/ontología-resultado; porque mientras esta sí puede apreciarse, aún si implícitamente, en la discusión bajo análisis, no me resulta tan claro que sea del todo operativa (o que lo sea del mismo modo) en el debate en la literatura metaontológica que he traído a colación. Finalmente $-\mathrm{y}$ en línea con el objetivo propuesto de contribuir a refinar la discusión en análisis y hacerla avanzar-, puede afirmarse que, incluso pudiendo reconstruirla como acerca de cuáles son las anclas fundamentales del derecho, todavía se podría, entonces, pasar a considerar cuestiones de fundamentación. En este sentido, seguramente sería imposible establecer relaciones de fundamentación entre hechos jurídicos de manera exhaustiva: ¡son demasiadas! Pero en cambio sería valioso, creo, establecer una tipología de relaciones de fundamentación entre (clases de) hechos jurídicamente distintivos, persistentes, ampliamente difundidos y - sobre todo— de alta fundamentalidad metafísica. Por lo demás - y así como ha sucedido con otras cuestiones metodológicas-, de los trabajos

47 Dejaré de lado aquí el modo preciso en que distinguir entre positivismos incluyente y excluyente, y lo que habría que considerar en el caso de que se sostenga que los hechos morales se encuentran fundamentados y/o anclados, a su turno, en hechos sociales. En todo caso, se aprecia que la discusión sobre la ontología jurídica podría resultar neutral a la disputa positivismo-antipositivismo, como ya se apuntaba en (Bernal Pulido, 2014b, pp. 148-150). 
primariamente enfocados como iusfilosóficos seguramente podrían surgir muy relevantes contribuciones también para la discusión metaontológica.

\section{Bibliografía}

Austin, J. (1995). The Province of Jurisprudence Determined, ed. W. Rumble. Cambridge: Cambridge University Press.

Bernal Pulido, C. (2014a). Austin, Hart y Shapiro. Tres variaciones alrededor del derecho como una entidad fundada en una práctica social, tr. M. Viana. Discusiones, XIV, 21-72.

Bernal Pulido, C. (2014b). Intencionalidad colectiva y estructura ontológica del derecho. Una respuesta a Richard Ekins y Damiano Canale, tr. H. Bouvier. Discusiones, XIV, 145-171.

Berto, F. \& Plebani, M. (2015). Ontology and Metaontology. A Contemporary Guide. London: Bloomsbury.

Blackburn, S. (1993). Essays in Quasi-Realism. Oxford: Oxford University Press. Bloomfield, P. (2009). Archimedeanism and Why Metaethics Matters. En R. Shafer-Landau, (ed.), Oxford Studies in Metaethics, IV (pp. 283-302). Oxford: Oxford University Press.

Bricker, P. (2006). The Relation Between the General and the Particular: Entailment vs. Supervenience. En D. Zimmerman (ed.), Oxford Studies in Metaphysics, II (pp. 251-288). Oxford: Oxford University Press.

Canale, D. (2014). ¿El derecho está fundado en la acción colectiva? Comentario al artículo de Carlos Bernal, tr. D. Domeniconi. Discusiones, XIV, 73-11.

Caracciolo, R. (1988). El sistema jurídico. Problemas actuales. Madrid: Centro de Estudios Constitucionales.

Chilovi, S. \& Pavlakos, G. (2019). Law-Determination as Grounding: A Common Grounding Framework for Jurisprudence. Legal Theory, 25, 53-76.

Clark, M. \& Liggins, D. (2012). Recent Work on Grounding. Analysis, 72(4), 812-823.

Dworkin, R. (2011). Justice for Hedgehogs. Cambridge: Harvard University Press.

Dworkin, R. (1996). Objectivity and Truth: You'd Better Believe It. Philosophy and Public Affairs, 25(2), 87-139. 
Pablo A. Rapetti

Ehremberg, K. (2008). Archimedean Metaethics Defended. Metaphilosophy, 39(4-5), 508-529.

Ekins, R. (2014). Hechos, razones y acción colectiva: reflexiones en torno a la ontología social del derecho, tr. N. Scavuzzo. Discusiones, XIV, 113-144.

Ekins, R. (2012). The Nature of Legislative Intent. Oxford: Oxford University Press.

Epstein, B. (2019). Anchoring versus Grounding: Reply to Schaffer. Philosophy and Phenomenological Research, 99, 768-781.

Epstein, B. (2015). The Ant Trap. Rebuilding the Foundations of the Social Sciences. Oxford: Oxford University Press.

Fine, K. (2012). A Guide to Ground. En F. Correia \& B. Schnieder (eds.), Metaphysical Grounding: Understanding the Structure of Reality (pp. 37-80). Cambridge: Cambridge University Press.

Fine, K. (2009). The Question of Ontology. En D. Chalmers, D. Manley \& R. Wasserman (eds.), Metametaphysics. New Essays on the Foundations of Ontology (pp. 157-177). Oxford: Oxford Clarendon Press.

Fine, K. (2001). The Question of Realism. Philosophers' Imprint, 1(1), 1-30. Gizbert-Studnicki, T. (2016). The Social Sources Thesis, Metepahysics and Metaphilosophy. En P. Banas, A. Dyrda \& T. Gizbert-Studnicki (eds.), Metaphilosophy of Law (pp. 121-146). Oxford: Hart Publishing.

Guastini, R. (2014). Otras distinciones, tr. D. Dei Vecchi et al. Bogotá: Universidad del Externado de Colombia.

Hart, H. (2000). Post scríptum al concepto de derecho, ed. J. Raz \& P. Bulloch, tr. R. Tamayo. Ciudad de México: UNAM.

Hart, H. (1982). Essays on Bentham. Oxford: Oxford University Press.

Hart, H. (1963). El concepto de derecho, tr. G. Carrió. Buenos Aires: Abeledo-Perrot.

Hart, H. (1958). Legal and Moral Obligation. En A. Melden (ed.), Essays in Moral Philosophy (pp. 82-107). Seattle: University of Washington Press.

Kelsen, H. (1979). Teoría pura del derecho, $2^{\circ}$ ed., tr. R. Vernengo. Ciudad de México: Porrúa.

Kramer, M. (2018). H.L.A. Hart. The Nature of Law. Cambridge: Polity Press. Ludwig, K. (2013). Proxy Agency in Collective Action. Noûs, 31(1), 75-105. Marmor, A. (2011). Philosophy of Law. Princeton: Princeton University Press.

Pettit, P. \& List, C. (2011). Group Agency. Oxford: Oxford University Press. 
Quine, W. (2002). Acerca de lo que hay. En Id., Desde un punto de vista lógico (pp. 39-60), $2^{\circ}$ ed., tr. M. Sacristán. Barcelona: Paidós.

Rapetti, P. (2019). Desacuerdos en el derecho y positivismo jurídico. Madrid: Marcial Pons.

Raz, J. (1999). Practical Reason and Norms (2a ed.). Oxford: Oxford University Press.

Rosen, G. (2010). Metaphysical Dependence: Grounding and Reduction. En B. Hale \& A. Hoffmann (eds.), Modality. Metaphysics, Logic and Epistemology (pp. 109-135). Oxford: Oxford University Press.

Roversi, C. (2018). Constitutive Rules and the Internal Point of View. Argumenta, 4(1), 139-156.

Schaffer, J. (2019). Anchoring as Grounding: On Epstein's the Ant Trap. Philosophy and Phenomenological Research, 99, 749-767.

Schaffer, J. (2009). On What Grounds What. En D. Chalmers, D. Manley \& R. Wasserman (eds.), Metametaphysics. New Essays on the Foundations of Ontology (pp. 347-383). Oxford: Oxford Clarendon Press.

Schroeder, M. (2008). Being For: Evaluating the Semantic Program of Expressivism. Oxford: Oxford University Press.

Searle, J. (2010). Making the Social World. The Structure of Human Civilization. Oxford: Oxford University Press.

Searle, J. (1992). Intencionalidad. Un ensayo en la filosofía de la mente (tr. E. Ujaldón). Madrid: Tecnos.

Shapiro, S. (2014). Legalidad, tr. D. Papayannis \& L. Ramírez. Madrid: Marcial Pons.

Thagard, P. (1978). The Best Explanation: Criteria for Theory Choice. Journal of Philosophy, 75(2), 76-92.

Toh, K. (2005). Hart's Expressivism and his Benthamite Project. Legal Theory, 1, 75-123.

Tuzet, G. (2014). Ontología social y práctica jurídica: un debate, tr. D. Dei Vecchi. Discusiones, XIV.

Von Wright, G. (1970). Norma y acción. Una investigación lógica (tr. P. García). Madrid: Tecnos.

Wilson, J. (2014). No Work for a Theory of Grounding. Inquiry, 57(5-6), 535-579. 